\title{
Desigualdades y vulnerabilidades en la condición juvenil: el desafío de la inclusión social
}

\author{
Inequalities and vulnerabilities among youths: the challenge \\ of social inclusion
}

\begin{abstract}
Alejandro José Capriati / alejandrocapriati@gmail.com
Universidad de Buenos Aires, Argentina
\end{abstract}

\begin{abstract}
The article presents a conceptual framework to address the social inclusion of adolescents and youths in a middle-income country like Argentina. The background of youths' social exclusion is the historical process of expropriation of welfare under the neoliberal state. I analyze social trajectories, situations of vulnerability and social and individual supports in heterogeneous settings of inequality. I also theoretically examine the ways of conceptualizing the social context and the constitution of the individual, facing institutional changes. In conclusion: it is necessary to investigate further the specific ways in which inequalities and vulnerabilities intersect, and to design technologies of intervention. These technologies should be based both on scientific evidence and the recognition of youths' rights.
\end{abstract}

Key words: youth, social inclusion, inequalities, vulnerability, supports.

Resumen: En el artículo planteo un marco conceptual para abordar algunas de las complejidades que el desafío de la inclusión social demanda, atendiendo especialmente a las problemáticas vinculadas con la adolescencia y la juventud. Las dificultades en cuanto a la inclusión social, asunto prioritario de la agenda en juventudes, tienen como trasfondo común un proceso histórico de expropiación del bienestar. Mi propuesta centra su foco de atención en las trayectorias sociales, las situaciones de vulnerabilidad y los soportes en escenarios heterogéneos de desigualdad. Asimismo, recupero nudos problemáticos de la teoría social relativos al modo de conceptualizar el contexto social y la constitución del individuo frente a las transformaciones institucionales. A modo de cierre, postulo la necesidad no sólo de investigar los modos específicos en que se intersectan desigualdades y vulnerabilidades, sino también de avanzar hacia el estudio y diseño de tecnologías de intervención, asentadas en evidencia y en compromiso con los derechos de los sujetos.

Palabras clave: juventudes, inclusión social, desigualdades, vulnerabilidades, soportes. 


\section{Introducción}

Cientistas sociales, organismos internacionales, gobiernos y medios de comunicación se preocupan por la cuestión juvenil (Silva Bega, 2013; Klisberg, 2010; Hopenhayn y Morán, 2008). Este fenómeno no está exento de ambigüedades: simplificando, pero no mucho, la situación de la juventud tiende a ser representada como un problema que debe ser controlado al mismo tiempo que se reconoce la necesidad de considerar a los jóvenes como actores estratégicos del desarrollo o se promueve un enfoque de derechos que reconoce su autonomía.

Dejando en suspenso el análisis de los discursos celebratorios o condenatorios de los estereotipos juveniles más recurrentes, la propuesta del artículo es establecer conexiones entre las transformaciones históricas, los contextos sociales y las prácticas juveniles, con el objeto de comprender cómo se anudan los pliegues de la desprotección y la vulnerabilidad en la adolescencia y juventud. Esta propuesta se inscribe dentro de la agenda de la cuestión juvenil en América Latina, marcada por la incertidumbre que generan los interrogantes en torno a los patrones de inclusión social, al tránsito hacia la vida autónoma, y a los modos de construir ciudadanía (Kliksberg, 2010; Bendit, Hann y Miranda, 2008; Hopenhayn y Morán, 2008; Pérez Islas, 2006).

La condición juvenil en tanto objeto de estudio condensa un nodo atravesado por la edad en su articulación con el género, la condición socioeconómica, la condición étnica, las culturas juveniles, entre otros clivajes de la vida social (Chaves, 2010, 2005; Urresti, 2007; Dayrell Juarez, 2003; Feixa, 1998; Reguillo, 2000; Bourdieu, 1990). Como se sabe, la edad es un dato necesario pero no suficiente: los procesos que se despliegan en la adolescencia y en el devenir joven, desde las transformaciones corporales hasta el acceso a la vida adulta, asumen diversos significados para varones y mujeres (y quienes se apartan del binarismo de género) en distintos contextos sociales y épocas históricas.

En el presente artículo planteo elementos conceptuales para abordar algunas de las complejidades que el desafío de la inclusión social demanda, atendiendo especialmente a las problemáticas vinculadas con la adolescencia y juventud.

En el primer apartado sintetizo algunas de las transformaciones acaecidas o aceleradas desde 1990, prestando especial atención a los modos en que han modificado las condiciones de vida y afectado los modos de vivir la adolescencia y juventud. En el segundo apartado articulo enfoques y concep- 
tos con el objeto de construir un marco conceptual capaz de comprender las vulnerabilidades y los soportes que operan en la condición juvenil, frente a las nuevas dinámicas de individuación y a las persistentes desigualdades estructurales. A modo de cierre, propongo estudiar los modos específicos en que se intersectan las desigualdades y vulnerabilidades para contribuir a la definición de estrategias y modos de intervención.

\section{Un escenario social en permanente transformación}

En la Argentina contemporánea, salvo el peso demográfico de adolescentes y jóvenes (entre 15 y 29 años), aproximadamente en torno a un cuarto de la población total en los últimos cuarenta años (Miranda et al., 2007; INDEC, 2010), pocas cosas han permanecido constantes a nivel nacional y global. Instituciones constitutivas de la vida social en general y del devenir joven en particular como la escuela, la familia, la política, el trabajo, afrontan cambios, tanto en su alcance y posibilidades como en sus normas y valores.

Los cambios en la sociedad argentina remiten a procesos sociales, económicos, políticos, culturales, que exigen dar cuenta de transformaciones globales y regionales del capitalismo contemporáneo. La imagen que los Estado-nación avanzados construyeron de sí mismos desde mediados del siglo $\mathrm{XX}$ como sociedades cada vez más democráticas gracias a la reducción de las desigualdades sociales (a cargo del Estado de Bienestar, en el caso de Europa, o vía efecto de derrame de la economía de mercado, en el caso de los Estados Unidos de Norteamérica), ha estallado ante el aumento de las privaciones y la multiplicación de protestas y tensiones étnicas en distintas regiones (Bourgois, 2010; Wacquant, 2007).

La globalización económica y el triunfo del individualismo son, recuperando el planteamiento de Fitoussi y Rosanvallon para el caso francés (2003), procesos determinantes del sentimiento de inseguridad e incertidumbre. Además de las desigualdades tradicionales o estructurales, vinculadas con la jerarquía de ingresos y acceso a la vivienda, aparecen nuevas desigualdades relativas a las condiciones de vida, de empleo, de salud y accesibilidad a servicios y equipamiento público (Fitoussi y Rosanvallon, 2003).

En los países de América Latina, el acceso a recursos y oportunidades está atravesado por marcadas desigualdades: por un lado, una porción más o menos reducida de jóvenes alcanza niveles de vida similares al segmento juvenil de los países industrializados; y por otro lado, la situación de la mayoría se asemeja a la de los países más pobres (CEPAL-OIJ, 2004, 2008). El acceso 
inequitativo sigue patrones en términos de segregación espacial, de clase, de género, orientación sexual y pertenencia étnica (Bendit et al., 2008). Así, los grupos juveniles con alto nivel económico y movilidad global tienden a parecerse más a grupos privilegiados de otras latitudes que a los jóvenes pobres de sus países (Krauskopf, 2008).

Los escenarios nacionales son heterogéneos en los países de la región y presentan diferencias al interior de cada uno de ellos. En las últimas dos décadas se han alternado ciclos de crisis y crecimiento económico, por un lado, y cambios en las orientaciones políticas de los gobiernos, por el otro. Las transformaciones vinculadas con reformas estructurales en el papel del Estado y en la orientación de las políticas macroeconómicas, agrupadas bajo la expresión modelo neoliberal, han profundizado la precarización en las condiciones de vida y afectado el ejercicio de derechos sociales en los cuales es necesaria la intervención del Estado para asegurarlos.

Las crisis económicas, políticas y sociales han inaugurado nuevos contextos en la región frente a la debacle neoliberal. En estos escenarios emergentes desde principios del siglo XXI se ha puesto en cuestión la capacidad de autorregulación de las fuerzas del mercado y se ha reconsiderado la intervención del Estado y sus dispositivos políticos e institucionales con el objeto de garantizar la equidad y promover la inclusión social (Aparicio Castillo, 2013).

De acuerdo con el Panorama Social de América Latina 2012 (CEPAL, 2012), la pobreza y la indigencia en la región continúan su tendencia descendente, situándose como una de las más bajas registradas en las últimas tres décadas, especialmente a partir del aumento de los ingresos en los hogares pobres. Pese a su reducción, la pobreza y la indigencia continúan en niveles altos (en el año 2011 el 29,4\% de la población de la región era pobre) y la desigualdad en materia de distribución de ingresos es uno de los principales desafíos en la región (Aparicio Castillo, 2013); si bien se constatan recuperación y crecimiento económico en la mayoría de los países de la región desde el año 2005, no ha resultado posible detener la expansión del mercado de trabajo informal y el acceso inequitativo a diversos bienes sociales.

En el caso argentino, los cambios iniciados hacia mediados de la década de 1970 y profundizados durante la década de 1990 implicaron un incremento de la desigualdad, una crisis en torno al empleo y una heterogeneización de la pobreza, transformando de modo significativo las condiciones de vida de la población joven (Epele, 2010; Svampa, 2005). Las altas tasas de desocupación en jóvenes y la creciente vulnerabilidad en las condiciones de 
empleo explican, en buena medida, por qué la población joven ha sido uno de los grupos más perjudicados por los cambios en la estructura ocupacional (Miranda et al., 2007).

El deterioro de las condiciones de vida hacia fines de la década de 1990 y primeros años del siglo XXI instalaron un "nuevo umbral" a partir del cual pensar las desigualdades en Argentina (Svampa, 2009). Estos cambios, presentados en sus trazos más generales, pueden ser leídos como una expropiación del bienestar, un proceso histórico con diversas etapas o periodos, no exento de contradicciones, que exige un análisis mayor tanto de sus continuidades como de sus rupturas.

Desde fines del año 2002, un periodo de crecimiento económico revirtió las altas tasas de desocupación e inició un ciclo de mejoras en distintos indicadores macroeconómicos y sociales, con controversias sobre diferentes indicadores desde 2007 o 2008. No resulta una ecuación simple precisar en qué medida se han revertido las brechas sociales abiertas en la década pasada, atendiendo al crecimiento económico y al impacto de las políticas sociales en materia de educación, empleo y transferencia de ingreso. Una de las hipótesis más fecundas para justipreciar el periodo 2003-2013 sostiene la existencia de tendencias contrapuestas en materia de igualdad-desigualdad a partir de un abordaje multidimensional de la desigualdad y diversas esferas de bienestar (Kessler, 2014).

Bajo esta hipótesis es posible identificar en materia de salud, por ejemplo, mejora de indicadores vitales y aumento de la cobertura de salud y persistencia de enfermedades ligadas a la pobreza. En el campo de los derechos sociales y especialmente en los derechos de niñas, niños y adolescentes ( $\mathrm{Tu}$ ñón, 2012), si bien ha sido positivo el avance en la creación de jurisprudencia es significativo el desafío de acortar la brecha institucional, es decir, reducir la distancia entre la sanción formal y la implementación de estrategias efectivas bajo la nueva jurisprudencia.

Las dificultades en cuanto a la inclusión social, definida como la posibilidad del ejercicio de la capacidad efectiva de acceder a los derechos civiles, económicos, sociales y culturales, tienen como trasfondo común un proceso de expropiación del bienestar. Este proceso, recuperando la definición de Epele (2010), apunta al resquebrajamiento de las formas tradicionales de bienestar, a la emergencia de promesas vinculadas al mercado y a la producción de nuevos malestares y padecimientos. 


\section{Una propuesta teórica para abordar el desafío de la inclusión social}

Los ejes analíticos que se proponen en el marco conceptual están atravesados por cambios históricos y refieren a distintos nudos problemáticos de la teoría social, entre los cuales se destaca la crisis de la representación unitaria de la sociedad y el modo en que definimos el contexto y abordamos la constitución del sujeto.

Cómo conceptualizar el lugar que le cabe en todo análisis social al contexto y a las posiciones sociales constituye, como desarrolla Martuccelli (2007a), uno de los problemas principales de la teoría social frente a la crisis de la idea del personaje social, es decir, la imposibilidad de explicar las acciones y experiencias de los individuos exclusivamente a partir de su posición social. El desfase entre las experiencias subjetivas y las procedencias colectivas dan cuenta del impasse teórico de las explicaciones que no atienden a la singularización creciente de las experiencias (Martuccelli, 2006). No se trata de sustraer el contexto del análisis social; el reto que propone Martuccelli es construir una teoría que reconozca el legítimo lugar del contexto y las posiciones sociales, y sea capaz de explicar la labilidad de ambas, focalizando en el entredós que se teje entre el actor y el sistema.

Situar al individuo en el centro del análisis no supone reducir el análisis al nivel del actor ni debe traducirse en la aceptación de una sociedad sin estructura. La individuación como estrategia de análisis, continuando con el planteamiento de Martuccelli (2006, 2007a), aborda el estudio de los principales cambios societales a partir del proceso de constitución del individuo y plantea como interrogante cómo se estructuran los fenómenos sociales a nivel de las experiencias personales, con el interés centrado en las consecuencias a escala de los actores de los cambios históricos.

Atendiendo a estas transformaciones, la propuesta para abordar el estudio de la condición juvenil está centrada en las trayectorias sociales, los procesos de vulnerabilidad y los soportes en escenarios heterogéneos de desigualdad. Las preguntas que dinamizan esta propuesta giran en torno de las relaciones entre trayectorias y escenarios, soportes y vulnerabilidades. ¿De qué maneras inciden los diferentes escenarios en la modulación de las trayectorias, en las situaciones de vulnerabilidad y en la disponibilidad de soportes? ¿Cuáles son las trayectorias juveniles típicas emergentes en los distintos escenarios de inclusión-exclusión? ¿Cuáles son las situaciones de vulnerabilidad que modelan las trayectorias y cuáles son los principales soportes que operan en las trayectorias? 
Este marco tiene tres ejes: caracterizar los escenarios de inclusión-exclusión, identificar situaciones de vulnerabilidad y construir una tipología de los soportes. Apunta a la especificidad de los problemas para comprender la concreción particular de las relaciones de género, de clase y generación, y captar cómo está siendo vivida la desigualdad en cada escenario social (Ayres et al., 2012a). Para ello se abordan el despliegue de los soportes y las situaciones de vulnerabilidad por medio de la noción de trayectoria social, en tanto modo más o menos típico de una persona o un grupo de transitar el espacio social, caracterizado por medio de la descripción del escenario social.

\section{a) Los escenarios sociales de inclusión-exclusión}

En este primer eje el punto de partida es pensar el usualmente denominado contexto (externo) como fenómeno (propiamente dicho). Los factores sociales no son "factores" ni son "externos"; por el contrario, determinan o producen al fenómeno en cuestión. Las desigualdades sociales no refieren a un conjunto agregado de desigualdades previas o exteriores, sino que son dimensiones integradas, no exentas de conflicto, que se retroalimentan y reproducen (Pecheny, 2013).

La noción de escenarios sociales es una forma de describir las posiciones estructurales y diferencias de oportunidad constreñidas a un área geográfica determinada, develando disparidades territoriales e inequidades socio-espaciales. En esta descripción se especifica el tipo de participación del Estado, del mercado y de la sociedad civil, prestando especial atención a los espacios de tipo comunitario, con el objeto de captar en cada escenario la matriz institucional y la accesibilidad a diversos bienes y servicios. La definición de accesibilidad está constitutivamente vinculada con el ejercicio de la ciudadanía e inclusión social (Hernández, 2012).

Se propone abordar los problemas de accesibilidad en su nivel territorial de máxima desagregación, tal como proponen Steinberg et al. (2011), para aprehender la estructura de oportunidades que en él se desarrolla. Por medio de este concepto se identifica la existencia de oferta y servicios en diversas áreas, desde educación y empleo, servicios básicos, cuidados a la infancia y centros de salud, hasta programas sociales y opciones en deporte y cultura (Steinberg et al., 2011).

En el Gran Buenos Aires, territorio formado por la Ciudad de Buenos Aires y veinticuatro partidos de la provincia de Buenos Aires, residen casi 13 millones de personas, de las cuales un tercio tienen entre 10 y 29 años de edad 
(INDEC, 2010). Las condiciones de vida y el acceso a recursos en una megalópolis como el Gran Buenos Aires develan múltiples desigualdades sociales (Tuñón, 2013; Steinberg, Cetrángolo y Gatto, 2011; Epele, 2010; Grassi y Danani, 2009; Bayón, 2008; Salvia, 2008; Malimacci y Salvia, 2005).

En el Gran Buenos Aires, incluso al interior de la mayoría de los partidos de la provincia de Buenos Aires, coexisten escenarios diversos de inclusiónexclusión. Por ejemplo, a pesar de la tendencia creciente de la matrícula escolar y de los años de escolaridad, son heterogéneas las posibilidades de acceso a una educación de calidad y su accesibilidad está condicionada por el sector social de pertenencia y lugar de residencia (Miranda, Otero y Corica, 2007). Por otro lado, el origen social constituye una variable medular en las posibilidades de inserción laboral de los jóvenes (Pérez, 2009). Además de intervenir indirectamente mediante el acceso a diferentes niveles de educación, incide directamente: a igual nivel educativo entre jóvenes de distinto origen social existen distintas tasas de empleo y desocupación, develando desventajas en las posibilidades de valorar las credenciales educativas (Pérez, 2009).

Además de la descripción de las diferencias de oportunidades constreñidas a un área geográfica determinada, otro insumo para caracterizar el escenario social son los perfiles socio- demográfico y epidemiológico, con especial atención en la población adolescente y joven, y la mortalidad por causas externas o muertes por violencias.

El término muertes por violencias, a diferencia de la noción de causa externa que implica un abordaje de tipo individual a imagen de las causas "naturales" de enfermedad, hace referencia a fenómenos complejos y problemas de salud colectiva, atendiendo a la magnitud de su impacto social (Alazraqui et al., 2012; Spinelli et al., 2011; Minayo, 2006). Para dar cuenta de la magnitud que asume la violencia en la vida de adolescentes y jóvenes, retomo la tendencia temporal de las muertes por violencia para la población entre 10 y 29 años de edad durante el periodo 1990-2010 en el territorio argentino (Spinelli et al., 2013).

En 1992 la tasa de mortalidad por agresiones era de 4,9 cada 100.000 habitantes; diez años más tarde, al iniciarse la crisis económica y política, la tasa se duplica y alcanza la máxima expresión del periodo (9,4 cada 100.000 habitantes en 2002). Las tasas de mortalidad por suicidios entre 1990- 2010 presentan guarismos relativamente similares a los registrados en las tasas de mortalidad por agresiones a lo largo del periodo (oscila entre 4,4 y 9,4 cada 100.000 habitantes); no obstante, una y otra se comportan de modo distinto: los suicidios presentan un crecimiento importante entre 1997 y 2003, y 
a partir de 2004 permanecen relativamente estables con tasas que duplican a las halladas al comienzo de la década de 1990. Los valores más elevados de la tasa de mortalidad por agresiones o por suicidios constituyen el piso de la tasa de mortalidad por lesiones en el tránsito. Estas tasas muestran marcadas diferencias por regiones y al interior del Gran Buenos Aires.

\section{b) El estudio de las vulnerabilidades y las violencias}

Apelo al enfoque de vulnerabilidad y derechos humanos parar observar cómo, en dónde y sobre qué sujetos o grupos se anudan los pliegues de la desprotección, la precariedad y la violencia en la adolescencia y juventud. En este enfoque, desarrollado de modo pionero en el campo de la salud a partir de la epidemia del VIH-SIDA (Mann et al., 1999), el objeto principal de atención se desplaza de las identidades, personales o sociales, hacia las relaciones sociales, base de las situaciones de vulnerabilidad, como las relaciones de género, las relaciones económicas y las relaciones generacionales (Ayres et al., 2012b). A diferencia de la noción de riesgo individual, la vulnerabilidad social pone el foco en el contexto o escenario en el cual se desarrollan los sujetos y las prácticas (Ayres et al., 2012a; Gruskin y Tarantola, 2012; Ayres et al., 2008).

El análisis de la vulnerabilidad es entendida como un conjunto de aspectos individuales y colectivos vinculados con una mayor susceptibilidad a padecer perjuicios y menor disponibilidad de recursos para su protección (Ayres et al., 2012b). Analíticamente, se distinguen tres dimensiones, en permanente interacción: lo individual, lo social y lo programático. Lo individual se refiere a las relaciones intersubjetivas, lo social a los espacios concretos de interacción, y lo programático a las políticas e instituciones. La definición de vulnerabilidad presenta una gran afinidad con la desigualdad entendida como dimensiones integradas que se retroalimentan y reproducen (Pecheny, 2013).

Las vulnerabilidades, así definidas, impactan sobre la dimensión de la salud, que a su vez, en el caso de los jóvenes, está indisolublemente ligada a situaciones de violencia (Capriati, 2013; Di Leo y Camarotti, 2013). Una definición amplia e integral de la salud reconoce como perteneciente a su campo problemático las prácticas, situaciones o condiciones capaces de producir tanto bienestar como malestar en los sujetos, grupos y comunidades (Kornblit, 2010).

El estudio de las vinculaciones entre juventudes, género y salud, especialmente en torno a las violencias y vulnerabilidades, es un área de desarro- 
llo incipiente, tanto desde las ciencias sociales como desde la salud pública (Pecheny, 2013; Di Leo et al., 2011; Kornblit, 2010; Epele, 2010; Reguillo, 2008; Ramos, 2006; Minayo 2006). Abordar el campo problemático de la salud juvenil en relación con la normatividad heterosexual, conlleva tematizar asuntos pocos explorados como los niños/as y jóvenes LGBT (lesbianas, gays, bisexuales y transexuales) y la especificidad de los procesos de vulneración en relación con los contextos estigmatizantes y/o violentos (Pecheny, 2013).

La condición de joven LGBT, más que yuxtaponerse, agrega dimensiones suplementarias que complejizan los procesos de vulnerabilidad social (Pecheny, 2013); la especificidad de la condición LGBT en tanto generación joven está vinculada no sólo a las enfermedades como el VIH/SIDA y otras ITS, sino también a situaciones de violencias y diversos padecimientos.

Además de las manifestaciones visibles de la violencia, se deben abordar las consecuencias de experiencias traumáticas crónicas como la pobreza, el clasismo y el sexismo, reconociendo los complejos vínculos entre procesos económicos-políticos y vida cotidiana (Epele, 2002). Específicamente, las nociones de cadena de violencia y cadena punitiva, emergentes de estudios realizados en el ámbito de la provincia de Buenos Aires, echan luz sobre las violencias interpersonales e institucionales. El concepto de cadena de violencia propone hacer inteligible cómo diferentes tipos de violencia se concatenan y dan forma a una cadena que conecta la calle y el hogar, el espacio doméstico, la esfera pública y las acciones del aparato estatal (Auyero y Berti, 2013). La noción de cadena punitiva se enfoca en las trayectorias marcadas por el encadenamiento de lo policial, lo judicial y lo custodial (Daroqui y López, 2012).

De manera específica, entre los aportes recientes al estudio de esta dimensión se destaca el análisis de los acontecimientos biográficos en la vida de varones y mujeres jóvenes residentes en villas y barrios populares del Gran Buenos Aires por medio de relatos de vida (Di Leo y Camarotti, 2013). Desde las perspectivas de los jóvenes, gran parte de los problemas que han vivenciado son de índole personal e intrafamiliar, asimilados en buena medida por medio de los vocabularios de la desgracia, del infortunio y del abuso (Martuccelli, 2013).

Desde una perspectiva enfocada en la vulnerabilidad social y los derechos humanos, las adversidades en la infancia y los problemas durante el devenir joven asumen otra forma y color: así, se devela la ausencia de espacios de cuidado, programas sociales o instituciones, que protejan o brinden asisten- 
cia para afrontar experiencias traumáticas como las privaciones crónicas, las agresiones familiares, las violencias cotidianas y los consumos problemáticos de drogas, reforzando la situación de desamparo en la temprana infancia o adolescencia (Capriati, 2014, 2013).

Los acontecimientos biográficos en las trayectorias de este grupo de jóvenes dan cuenta de un nivel alto de vulnerabilidad social, pautado no sólo por la mayor susceptibilidad de perjuicios, sino también por la menor disponibilidad de recursos para su protección y por la ausencia de instancias oportunas de reparación. No debiera ser necesario aclarar que el enfoque en la vulnerabilidad no significa que una conducta de riesgo carezca de consecuencias o importancia (Parker y Aggleton, 2003); es una perspectiva más amplia que considera tanto las prácticas de las personas y sus relaciones sociales como el impacto del Estado, por acción u omisión (Pecheny, 2013).

La heterogeneidad de las condiciones de vida y de los procesos de subjetivación, retomando el planteo de Di Leo (2011), hace que las violencias sean vivenciadas de diferentes formas por distintos grupos de jóvenes, dependiendo no sólo de la posición en el espacio social, sino también de las condiciones de género, la utilización de recursos o capitales, y la apropiación de derechos, entre otros aspectos, que son aprehendidos a partir de la noción de soporte, tercer eje analítico.

\section{c) El estudio de los soportes}

Para el análisis de las maneras por las cuales las mujeres y varones jóvenes se sostienen en el mundo, apelo a la noción de soporte, tal como ha sido desarrollada por Martuccelli (2007a, 2007b). No hay individuos sin soportes y no todos los soportes permiten la constitución del individuo. Los soportes son entendidos como medios materiales e inmateriales, relaciones u objetos, experiencias o actividades diversas, que permiten a los individuos sostenerse en la existencia.

La noción de soporte en su concepción ampliada se muestra como una herramienta de análisis productiva para comprender el diferencial de maneras por las cuales los individuos se sostienen en el mundo (Martuccelli, 2007b). A diferencia de las concepciones tradicionales del término, vinculadas con las condiciones socioeconómicas, los derechos sociales y el sistema de protección (Castel, 1997), este abordaje ampliado abre el análisis a las dimensiones sociales y existenciales, y deja abierto el carácter de los soportes, no definiendo a priori cuáles son los elementos susceptibles de jugar ese papel 
(Martuccelli, 2007b). Por supuesto, los derechos y recursos económicos son soportes mayores del individuo, pero no son necesariamente los más importantes ni los únicos existentes.

A diferencia de los recursos o capitales, los soportes rara vez son utilizados a nivel instrumental, son de tipo relacional, escapan al control unilateral y suponen vínculos intersubjetivos (Martuccelli, 2006). El análisis de los soportes, sean éstos del orden afectivo, material o simbólico, revela que tienen distintas legitimidades y que no siempre poseen la capacidad de funcionar como amortiguador social ante situaciones adversas o de crisis.

Una de las dificultades en el estudio de los soportes es la representación del individuo como autorrealización, representación heroica y dominante de la cultura occidental, ilusión de un sujeto no dependiente (Martucce1li, 2007b). Además de tener visibilidades diferentes, los soportes también tienen legitimidades distintas, invisibles, estigmatizantes, ambivalentes y patologizantes. Así, personas y grupos en posición de privilegio (social, económico, cultural) tienden a poseer soportes invisibles, incrementando el sentimiento de un sujeto que se sostiene y realiza efectivamente desde el interior. Por el contrario, los individuos en situación de fragilidad social, obligados a sostenerse en mayor medida desde el interior, paradójicamente son definidos como dependientes, en tanto sus soportes están vinculados con programas públicos.

Tal como explica Di Leo et al. (2011), el desarrollo de Martuccelli de una sociología de los soportes implica una ruptura epistemológica con la concepción dominante del individuo moderno, ya sea respecto a la imagen del individuo que se sostiene desde el interior o al imperativo del individuo soberano, figuras talladas a partir de los atributos de independencia y autonomía.

Recuperando la investigación sobre jóvenes del Gran Buenos Aires (Capriati, 2014), en los relatos aparecen diversos espacios que han desempeñado papeles reconocidos como positivos en sus biografías. Espacios heterogéneos, vinculados con un centro comunitario, con un bachillerato popular o una parroquia, que les permiten reflexionar sobre sí mismos y su futuro, que les habilita la posibilidad de viajar por fuera de su localidad, que les facilita continuar y finalizar sus estudios.

En cierta medida, estos espacios que funcionan como soportes pueden ser adjetivados como conmutadores, adaptando el uso de Ana Lía Kornblit (2010), en tanto ponen en circulación otros discursos y actúan como plataformas que posibilitan el ejercicio de la autonomía y promueven el desarrollo 
de proyectos biográficos. En la elaboración de un marco conceptual sobre promoción de la salud entre jóvenes, Kornblit (2010) retoma la noción de shifters o conmutadores, utilizada previamente por Michel de Certeau para captar la capacidad de ciertas personas o instituciones de relativizar ciertos saberes y poner en circulación otros discursos; la elaboración original del término remite a la lingüística de Jakobson. La inclusión de los espacios grupales entendidos como conmutadores en relación con la promoción de la salud obedece a que actúan "como plataformas que posibilitan el ejercicio de la autonomía”, pilar de una concepción integral de la salud.

\section{A modo de cierre}

Las antinomias, heredadas del proceso formativo de las ciencias sociales, relativas a comunidad- sociedad, estructura social-acción social, consensoconflicto, estructura-infraestructura, y particularmente, individuo-sociedad, se han visto desafiadas desde mediados de los años setenta, con importantes implicancias a nivel del individuo (Charry y Rojas Pedemonte, 2013).

En el caso de los actores juveniles, las instituciones que habían sido condiciones de su surgimiento durante el siglo XX afrontan procesos de re-configuración frente a un mundo social que cambia y torna obsoleto el modo como se definía la familia, la escuela, la pareja, el empleo, etcétera. A diferencia de tres o cuatro décadas atrás dependiendo de lugares, a principios del siglo XXI las instituciones ofrecen menos bases de apoyo capaces de organizar los tiempos sociales bajo la forma de la previsibilidad (Kessler y Merklen, 2013) y las dinámicas de individuación cobran especial interés (Araujo y Martuccelli, 2012; Martuccelli, 2010; Castel, 1997).

La orientación cada vez mayor de las transformaciones institucionales hacia el individuo obliga a que cada persona desarrolle su propia trayectoria biográfica (Martuccelli, 2006) y plantea el interrogante sobre los nuevos imperativos que pesan sobre los sujetos. Así, junto a los mayores espacios de autonomía e independencia vinculados con las demandas sociales de libertad individual emergen renovadas exigencias al individuo de hacerse a sí mismo (Merklen, 2013); requerimientos que en contextos de profundas desigualdades instauran como efectos paradójicos nuevas formas de control social; especialmente atendiendo al hecho de que el individualismo entre los sectores populares se conjuga con la inestabilidad y las exigencias de responsabilización que las nuevas configuraciones institucionales promueven (Kessler y Merklen, 2013). 
En razón de estos procesos, el marco conceptual elaborado procura evitar en el estudio de la condición juvenil tanto el determinismo social como la ilusión del sujeto libre y autorrealizado. La propuesta a lo largo del artículo ha sido articular enfoques y conceptos que permitan establecer conexiones entre los cambios históricos, los contextos sociales y las prácticas juveniles con el objeto de inscribir el estudio de la condición juvenil y la problemática de la inclusión social en un proceso de expropiación del bienestar, así como también en un escenario signado por los cambios en las relaciones entre individuo-sociedad.

Específicamente, el marco conceptual desarrollado está centrado en estudiar empíricamente cómo los procesos de vulnerabilidad y los soportes se ponen en relación en diferentes escenarios de desigualdad. Como se ha explicitado, existe gran afinidad entre la desigualdad y la vulnerabilidad en tanto procesos que se retroalimentan. Por un lado, la noción de escenarios sociales capta las posiciones estructurales y diferencias de oportunidad constreñidas a un área determinada. Por otro lado, el enfoque de vulnerabilidad sitúa el eje en las relaciones sociales (de género, socioeconómicas, étnicas, generacionales, etc.), base productora de las situaciones de vulnerabilidad, negligencia y violación de los derechos humanos (Ayres et al., 2012a).

Finalmente, el estudio de los soportes, en el sentido de Martuccelli (2007), propone aprehender las capacidades existenciales y sociales del individuo de sostenerse en el mundo y comprender cómo se insertan en entornos sociales y existenciales singulares, inscribiendo las historias de los individuos en entramados particulares de interdependencias.

El estudio de las situaciones de vulnerabilidad y los soportes en distintas trayectorias juveniles y escenarios sociales pone de relieve desafíos que enfrentan no sólo la investigación social sino también las políticas públicas que promueven la inclusión social frente a las transformaciones en las sociedades contemporáneas.

Como es sabido, los escenarios de desigualdad y las situaciones de vulnerabilidad socavan de modos diversos y con disímil intensidad las condiciones, materiales y simbólicas, que garantizan el acceso a los derechos sociales, económicos y culturales, consagrados en términos formales, promovidos en políticas públicas asentadas en la noción de expansión de derechos.

No es muy novedoso afirmar que las desigualdades, de tipo socioeconómico, de género, sexuales, entre otras, cuando son altas y persistentes como en América Latina, impactan de modo significativo en la vida de las personas más vulnerables (Pecheny, 2013). El impacto no es exterior, sino constituti- 
vo: las desigualdades producen tales vulnerabilidades; en otras palabras, las vulnerabilidades no son resultado de actitudes individuales o familiares, de conocimientos y prácticas, remiten a procesos estructurales. Lo novedoso, coincidiendo con Pecheny (2013), es identificar patrones específicos de relaciones con el objeto de intervenir, es decir, un doble desafío. Por un lado, dar cuenta empíricamente de las producciones diferenciales de vulnerabilidad generacional, atendiendo a la clase, al género, a la orientación sexual, a la etnicidad, entre otros clivajes sociales. Por otro lado, avanzar en el estudio y diseño de tecnologías de intervención que aborden estos problemas y procesos.

Dichos desafíos conllevan un salto e implican volver a preguntarnos acerca de los aportes y límites de la investigación: ¿̇esde dónde y cómo intervenir desde la política pública? Las distintas disciplinas científicas pueden pensar y proponer tecnologías de intervención para políticas de promoción de la salud, asentadas en evidencia y en compromiso con los derechos de los sujetos y los diversos proyectos subjetivos de felicidad (Pecheny, 2013; Ayres et al., 2012a).

Para ello, en el análisis de las trayectorias de individuos y grupos se debe discutir las experiencias que producen una política de expansión de derechos, interrogando en qué medida puede corresponder en sus intervenciones y efectos a la tensión entre proyectos autonomistas y nuevos controles (Silva Bega, 2013). La tensión entre autonomía y control social constituye una clave de lectura fundamental en el abordaje de la inclusión social como proceso.

No se trata de que adolescentes y jóvenes se integren a un orden social dado, reconozcan los valores culturales y adquieran ciertas competencias funcionales, los desafíos que la inclusión social pone de relieve refieren a la accesibilidad a oportunidades y recursos, a las condiciones materiales y simbólicas para ejercer los derechos. Tener como clave de lectura la tensión entre autonomía y control social significa, entre otras cosas, no dejar de preguntarse en qué medida las políticas, estrategias e intervenciones favorecen el desarrollo de un pensamiento crítico, contribuyen a la ampliación del universo sociocultural, generan las condiciones para que cada individuo sea dueño de su propia vida.

Tales preguntas resultan especialmente vigentes cuando se postula como prioritaria la cuestión de cómo garantizar el desarrollo pleno en adolescentes y jóvenes, el acceso a la educación, al empleo y a la cultura, el derecho a la salud integral, sin discriminaciones negativas de ningún tipo.

En síntesis, el interés central de este artículo ha radicado en presentar un marco conceptual y algunos interrogantes teóricos que contribuyan a la defi- 
nición de una agenda de investigación en juventudes, que apunte no sólo a la descripción y elaboración de teorías sino también a la necesidad de avanzar hacia el estudio de tecnologías de intervención.

\section{Bibliografía}

Alazrazqui, Marcio et al. (2012), "Calidad de los sistemas de información de mortalidad por violencias en Argentina y Brasil - 1990-2010”, en Ciência \& Saúde Coletiva, vol. 17, núm. 12, Brasil: Abrasco.

Aparicio Castillo, Pablo Christian (2013), "Educar y trabajar en contextos de precariedad y desigualdad en América Latina. Jóvenes en debate", en Revista Latinoamericana de Ciencias Sociales, Niñez y Juventud, año 11, núm. 2, Colombia: Universidad de Manizales.

Araujo Kathya y Danilo Martuccelli (2012), Desafios comunes. Retrato de la sociedad chilena y sus individuos, Chile: LOM.

Auyero, Javier y María Fernanda Berti (2013), La violencia en los márgenes. Una maestra y un sociólogo en el conurbano bonaerense, Argentina: Katz Editores.

Ayres, Jose Ricardo et al. (2008), "El concepto de vulnerabilidad y las prácticas de salud: nuevas perspectivas y desafíos", en Czeresnia, Dina y Carlos Machado [orgs.], Promoción de la salud. Conceptos, reflexiones y tendências, Buenos Aires: Lugar Editorial.

Ayres, Jose Ricardo et al. (2012a), "Conceitos e práticas de prevenção: da história natural da doença ao quadro da vulnerabilidade e direitos humanos", en Paiva, Vera et al. [orgs.], Vulnerabilidade e direitos humanos. Prevenção e promoção de saúde, Livro 1, Brasil: Juruá Editora.

Ayres, Jose Ricardo et al. (2012b), "Direitos humanos e vulnerabilidade na prevenção e promoção de saúde: uma introdução", en Paiva Vera et al. [orgs.], Vulnerabilidade e direitos humanos. Prevenção e promoção de saúde, Livro 1, Brasil: Juruá Editora.

Bayón, María Cristina (2008), "Desigualdad y procesos de exclusión social. Concentración socioespacial de desventajas en el Gran Buenos Aires y la Ciudad de México", en Estudios Demográficos y Urbanos, vol. 23, núm. 1, México: El Colegio de México.

Bendit, Rene et al. (2008), Los jóvenes y el futuro. Procesos de inclusión social y patrones de vulnerabilidad en un mundo globalizado, Argentina: Prometeo.

Bourdieu, Pierre (1990), "La juventud no es más que una palabra", en Sociología y Cultura, México: Grijalbo.

Bourgois, Philippe (2010), En busca de respeto, Argentina: Siglo XXI.

Capriati, Alejandro José (2013), “'Cómo salir del barrio sin morir en el intento’: trayectorias juveniles y proyectos de vida", en Di Leo Pablo Francisco y Ana Clara Camarotti [editores] "Quiero escribir mi historia". Vidas de jóvenes en barrios populares, Argentina: Biblos.

Capriati, Alejandro José (2014), “Una aventura abierta': acontecimientos biográficos de jóvenes residentes en villas y barrios populares del Gran Buenos Aires, Argentina”, en Ultima Década, núm. 40, Chile: CIDPA. 
Alejandro José Capriati. Desigualdades y vulnerabilidades en la condición juvenil: el desafío de la inclusión social

Castel, Robert (1997), La metamorfosis de la cuestión social. Una crónica del asalariado, Argentina: Paidós.

CEPAL-OIJ (2004), La Juventud en Iberoamérica: tendencias y urgencias, Santiago de Chile: Naciones Unidas.

CEPAL-OIJ (2008), Juventud y cohesión social en Iberoamérica. Un modelo para amar, Santiago de Chile: Naciones Unidas.

Charry, Carlos Andrés y Nicolás Rojas Pedemonte (2013), La era de los individuos: Actores, politica y teoría en la sociedad actual, Santiago: LOM.

Chaves, Mariana (2005), "Juventud negada y negativizada: representaciones y formaciones discursivas vigentes en la Argentina contemporánea”, en Última Década, núm. 23, Chile: CIDPA.

Chaves, Mariana (2010), Jóvenes, territorios y complicidades. Una antropología de la juventud urbana, Argentina: Espacio.

Daroqui, Alcira y Ana Laura López (2013), “La cadena punitiva: actores, discursos y prácticas enlazadas”, en Daroqui Alcira, López Ana Laura y García Roberto Félix [coords.], Sujetos de castigos. Hacia una sociología de la penalidad juvenil, Rosario: Homo Sapiens.

Dayrell Juarez, Tarcísio (2003), “O jovem como sujeito social”, en Revista Brasileira de Educação, vol. 51, núm. 24, Brasil: ANPEd.

Di Leo, Pablo Francisco y Ana Clara Camarotti [editores] (2013), "Quiero escribir mi historia". Vidas de jóvenes en barrios populares, Argentina: Biblos.

Di Leo, Pablo Francisco et al. (2011), "Procesos de individuación y relatos biográficos: articulaciones y potencialidades para el abordaje de experiencias juveniles en el campo de la promoción de la salud", en IX Jornadas de Sociología - Pre ALAS Recife, Ciudad Autónoma de Buenos Aires: Facultad de Ciencias Sociales (UBA).

Epele, María Esther (2002), "Violencia y trauma. Políticas del sufrimiento social entre usuarias de drogas”, en Cuadernos de Antropología Social, núm. 14, Argentina: Facultad de Filosofía y Letras (UBA).

Epele, María Esther (2010), Sujetar por la herida. Una etnografía sobre drogas, pobreza y salud, Argentina: Paidós.

Feixa, Carles (1998), De jóvenes, bandas y tribus. Antropología de la juventud, Barcelona: Ariel.

Fitoussi, Jean Paul y Pierre Rosanvallon (2003), La nueva era de las desigualdades, Buenos Aires: Manantial.

Grassi, Estela y Claudia Danani (2009), El mundo del trabajo y los caminos de la vida: trabajar para vivir, vivir para trabajar, Argentina: Espacio Editorial.

Gruskin, Sofia y Daniel Tarantola (2012), “Un panorama sobre saúde e directos humanos”, en Paiva Vera et al. [orgs.], Vulnerabilidade e direitos humanos. Prevenção e promoção de saúde, Livro 1, Brasil: Juruá Editora.

Hernández, Diego (2012), “Activos y estructuras de oportunidades de movilidad. Una propuesta analítica para el estudio de la accesibilidad por transporte público, el bienestar y la equidad", en EURE, vol. 38, núm. 115, Chile: Universidad Católica de Chile. 
Hopenhayn, Martín y María Luz Morán (2008), "Miradas cruzadas sobre la juventud en Iberoamérica”, en Pensamiento Iberoamericano, núm. 3, vol. XIII-XXIX, España: CEPAL y Universidad Complutense de Madrid.

Kessler, Gabriel (2014), Controversias sobre la desigualdad: Argentina, 2003-2013, Buenos Aires: Fondo de Cultura Económica.

Kessler, Gabriel y Denis Merklen (2013), “Una introducción cruzando el Atlántico”, en Castel, Robert et al., Individuación, precariedad, inseguridad, Argentina: Paidós.

Kliksberg, Bernardo (2010), Es difícil ser joven en América Latina, Buenos Aires: Sudamericana.

Kornblit, Ana Lía (2010), "La promoción de la salud entre los jóvenes”, en Acta Psiquiatr Psicol Am Lat., vol. 56, núm. 3, Argentina: Fundación ACTA.

Krauskopf, Dina (2008), “Dimensiones de la participación en las juventudes contemporáneas latinoamericanas”, en Revista Pensamiento Iberoamericano, vol. 3, XIII-XXIX, España: CEPAL y Universidad Complutense de Madrid.

Malimacci, Fortunato y Agustín Salvia (2005), Los nuevos rostros de la marginalidad urbana, Buenos Aires: Biblos.

Martuccelli, Danilo (2006), Forgé par l'épreuve. L'individu dans la France contemporaine, París: Armand Colin.

Martuccelli, Danilo (2007a), Gramáticas del individuo, Argentina: Losada.

Martuccelli, Danilo (2007b), Cambio de rumbo. La sociedad a escala del individuo, Santiago de Chile: LOM.

Martuccelli, Danilo (2013), "Prefacio”, en Di Leo Pablo Francisco y Camarotti Ana Clara [edit.], Quiero escribir mi historia. Vidas de jóvenes en barrios populares, Argentina: Biblos.

Minayo, María Cecilia (2006), Violência e Saúde, Brasil: Editora Fiocruz.

Mann, Jonathan et al. [eds.] (1999), Health and Human Rights: A Reader, New York: Routledge.

Merklen, Denis (2013), “Las dinámicas contemporáneas de la individuación”, en Castel, Robert et al., Individuación, precariedad, inseguridad, Argentina: Paidós.

Miranda, Ana et al. (2007), "Cambio y situación social de los jóvenes en Argentina”, en Papeles de Población, vol. 13, núm. 52, México: Universidad Autónoma del Estado de México.

Parker, Richard y Peter Aggleton (2003), "HIV and AIDS-related stigma and discrimination: a conceptual framework and implications for action", en Social Science and Medicine, vol. 57, USA: Elsevier.

Pecheny, Mario Martín (2013), "Desigualdades estructurales, salud de jóvenes LGBT y lagunas de conocimiento: ¿qué sabemos y qué preguntamos?”, en Temas de Psicología, vol. 21, núm. 2, Brasil: Sociedade Brasileira de Psicologia.

Pérez Islas, José Antonio (2006), “Trazos para un mapa de la investigación sobre juventud en América Latina”, en Papers. Revista de Sociología, vol. 79, España: Universidad Autónoma de Barcelona y REDI. 
Ramos, Silvia (2006), "Brazilian responses to violence and new forms of mediation: the case of the Grupo Cultural AfroReggae and the experience of the project 'Youth and the Police'”, en Ciência. saúde coletiva, vol. 11, núm. 2, Brasil: ABRASCO.

Reguillo, Rossana (2000), Emergencia de culturas juveniles. Estrategias del Desencanto, Argentina: Grupo Editorial Norma.

Reguillo, Rossana (2008), “Las múltiples fronteras de la violencia: jóvenes latinoamericanos entre la precarización y el desencanto", en Pensamiento Iberoamericano, vol. 3, XIIIXXIX, España: CEPAL y Universidad Complutense de Madrid.

Salvia, Agustín (2008), Jóvenes promesas. Trabajo, educación y exclusión social en la Argentina, Argentina: Miño y Dávila.

Silva Bega, Maria Tarcisa (2013), "Políticas públicas para juventude: normatização e controle social”, en XXIX Congreso ALAS Chile, del 29 de septiembre al 4 de octubre.

Spinelli, Hugo et al. (2013), "Vulnerabilidad y derechos sociales: datos y notas sobre mortalidad por violencias en adolescentes y jóvenes en Argentina (1990-2010)”, en Alvarado, Arturo [comp.], Mortalidad en América Latina, México: Colegio de México. [Capítulo aprobado por el Comité Editorial del Colegio de México, en prensa.]

Spinelli, Hugo et al. (2011), Mortalidad por armas de fuego en Argentina, 1990-2008, Argentina: Organización Panamericana de la Salud (OPS).

Svampa, Maristela (2005), La sociedad excluyente. La Argentina bajo elsigno delneoliberalismo, Argentina: Taurus.

Svampa, Maristela (2009), Cambio de época: movimientos sociales y poder politico, Argentina: Siglo XXI.

Urresti, Marcelo (2007), "De la cultura del aguante a la cultura del reviente", en Margulis, Mario et al. [comp.], Familia, habitat, y sexualidad en Buenos Aires. Investigaciones desde la dimensión cultural, Buenos Aires: Biblos.

Wacquant, Loic (2007), Los condenados de la ciudad. Guetos, periferias y Estado, Buenos Aires: Siglo XXI.

\section{Recursos electrónicos}

CEPAL (2012), Panorama social de América Latina. Disponible en: http://www.eclac. org/publicaciones/xml/5/48455/PanoramaSocial2012DocI-Rev.pdf (13 de febrero 2013).

INDEC (2010), Censo Nacional de Población, Hogares y Viviendas 2010. Disponible en: http://www.censo2010.indec.gov.ar/preliminares/cuadro_24.asp (23 de febrero 2011).

Pérez, Pablo (2009), "Desigualdad social e inserción laboral de jóvenes en el periodo posconvertibilidad”, en Novedades CEIL, núm. 3. Disponible en: http://www.ceilconicet.gov.ar/divulgacion/articulo-del-mes/desigualdad-social-e-insercion-laboralde-jovenes-en-el-periodo-posconvertibilidad-pablo-perez/ (22 de agosto de 2014).

Steinberg, Cora et al. (2011), "Desigualdades territoriales en la Argentina. Insumos para el planeamiento estratégico del sector educativo”, en Documentos de proyectos, núm. 53, 
CEPAL, UPEA. Disponible en: http://www.cepal.org/publicaciones/xml/8/43168/ DocW53fin.pdf (01 de noviembre de 2012).

Tuñón, Ianina (2013), "Inclusión educativa y estructura de oportunidades para la recreación. Niñez y adolescencia en la Argentina urbana", en Observatorio de la Deuda Social Argentina, vol. 1. Disponible en: http://www.uca.edu.ar/uca/common/grupo81/ files/Boletin_UCARecreacion.pdf (20 de agosto de 2013).

Alejandro J. Capriati. Lic. en Sociología y Doctor en Ciencias Sociales por la Universidad de Buenos Aires. Docente de la Facultad de Ciencias Sociales (UBA) y becario posdoctoral (CONICET) en el Instituto de Investigaciones Gino Germani (UBA). Líneas de investigación: estudios sobre juventudes y vulnerabilidad, salud, género y derechos. Publicaciones recientes: "'Una aventura abierta': acontecimientos biográficos de jóvenes residentes en villas y barrios populares del Gran Buenos Aires, Argentina”, en Última Década, núm. 40, Chile: CIDPA (2014), disponible en: http://www.cidpa. $\mathrm{cl} /$ ? $\mathrm{p}=1362$ (22 de agosto de 2014); "La condición juvenil y las iniciativas culturales barriales en el Gran Buenos Aires", en Question. Revista especializada en Periodismo y Comunicación, vol. 1, núm. 40 (2013), disponible en: http:// perio.unlp.edu.ar/ojs/index.php/question/article/viewFile/1968/1718 (22 de agosto de 2014); con Mónica Petracci, Mario Pecheny y Marina Mattioli, "El aborto en las trayectorias de mujeres y varones de la ciudad de Buenos Aires”, en Sex., Salud Soc. (2012), disponible en: http://www.scielo.br/scielo.php?script $=$ sci_arttext\&pid=S1984-64872012000600008 (22 de agosto de 2014).

Recepción: 22 de agosto de 2014.

Aprobación: 29 de junio de 2015. 